\title{
Image Analysis of EDS and Backscatter SEM Images of Pharmaceutical Tablets
}

\author{
R. A. Carlton
}

GlaxoSmithKline, King of Prussia, PA 19406

Pharmaceutical tablets are composed of a number of different materials, each of which is designed to improve performance. The drug substance (active pharmaceutical ingredient API) is intended to act on the disease state or the symptoms of the disease. The other components (referred to as excipients) act as fillers, bulking agents, tablet disintegrants, tablet coatings (to protect the core and to mask taste) and as lubricants for the tablet die among other purposes. Consistent performance of the tablets depends directly upon the amount of each excipient in the tablet. The choice of excipient and the amounts of each are the subject of intense study in pharmaceutical development. The most common measure of tablet performance is tablet dissolution testing. Although this type of test is valuable for assessing overall performance, it is useful to have means to investigate the distribution of excipients and API within the tablet itself. The use of EDS maps and BSE images of tablet cross-sections are two related means of directly examining excipient and API distribution within a tablet.

While some work has been reported in this field $[1,2,3]$, the emphasis has been on the qualitative assessment of excipient and API distribution. It is clearly desirable to move to more quantitative measures of distribution using image analysis. Full method development and error analysis will require extended study of sample preparation, sampling statistics, size and shape parameters, and precision. This poster presents some initial results in the development of quantitative methods using a multi-vitamin tablet as a test case.

A Hitachi $3500 \mathrm{~N}$ variable pressure SEM was used for the backscatter imaging and Clemex Vision PE 5.0 was used for the image analysis. A Bruker $30 \mathrm{~mm}^{2}$ area SDD detector was used to collect the EDS maps. Figure 1 presents a typical backscatter image of the tablet cross-section along with the accompanying EDS map. This particular map combines colors in one although the individual element maps were used for analysis. Figure 2 presents the relative area percentages of the different gray level regions in the image. The percentages represent the area of each gray level region of interest in relation to the total field area. Twenty images were collected and measured.

Each of the two imaging modes provides advantages and disadvantages. The backscatter image can be collected quickly and provides an average atomic number contrast. Consequently, gray level regions of interest accurately reflect molecular composition which is the item of interest. On the other hand, one must do the EDS beforehand to know what molecule is represented by each field of interest. EDS maps take longer to collect, even with SDD detectors, and are reflective of atom composition. On the other hand, there is little ambiguity concerning the chemical nature of each region. The image analysis results can be presented either as field or as object data. Figure 2 presents field data, but it is interesting to assess the shape and size of different molecules. For instance, the Si rich region is stringy and exists in grain boundary regions whereas the S rich regions are nearly circular regions. Numerical values for these shape factors can aid in understanding the distribution of the chemicals in the tablet. 


\section{REFERENCES}

[1] Vogt A.D., et al Microscopy and Microanalysis, 15 (Suppl 2) 2009, pp 380 - 1.

[2] Neilly J.P., et al Microscopy and Microanalysis, 15 (Suppl 2) 2009, pp 18-19.

[3] Carlton R.A., Microscopy and Microanalysis, 15 (Suppl 2) 2009, pp 380 - 1.

\section{ACKNOWLEDGEMENTS}

My thanks to Ted Juzwak of Bruker for help with the silicon drift detector EDS maps.
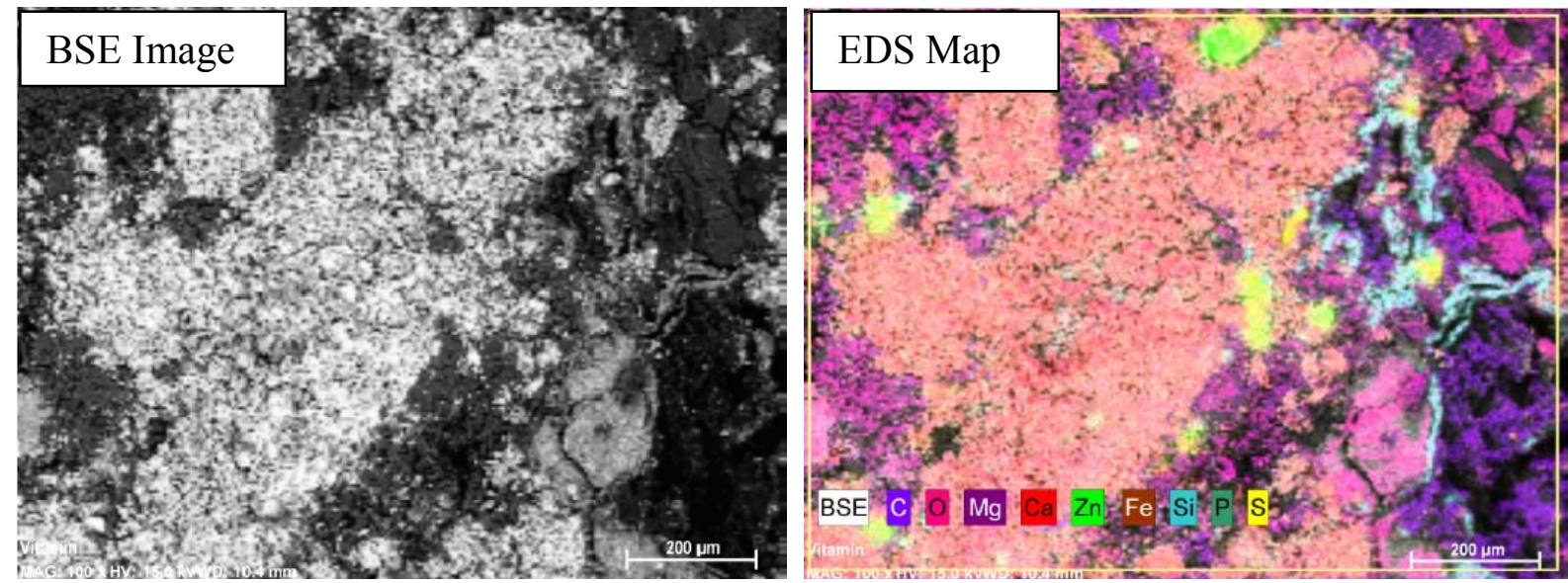

FIG. 1 Backscatter electron image and EDS map of typical cross-section of multi-vitamin.

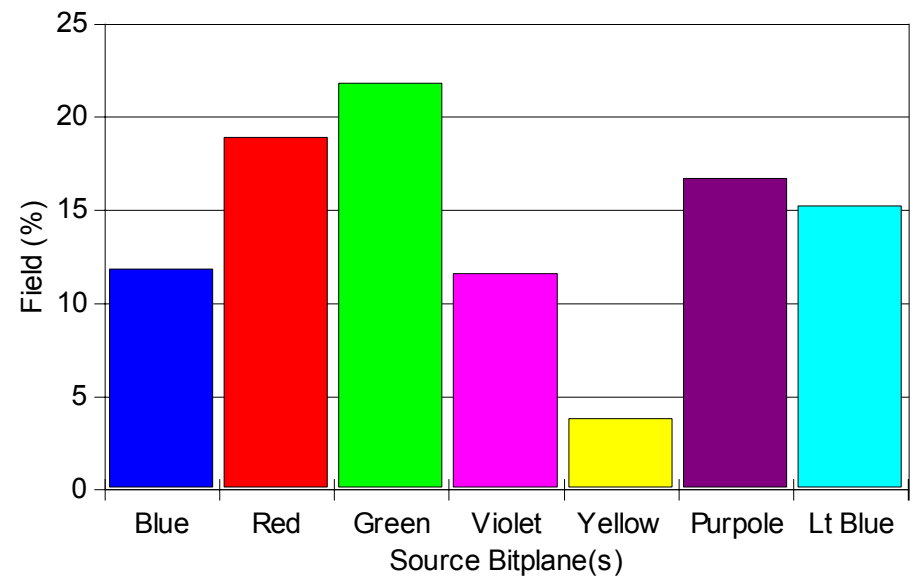

\begin{tabular}{|ll|}
\hline Region of Interest & Field (\%) \\
Blue & 11.87 \\
Red & 18.94 \\
Green & 21.83 \\
Violet & 11.60 \\
Yellow & 3.83 \\
Purple & 16.70 \\
Lt Blue & 15.23 \\
Total & $\mathbf{1 0 0}$ \\
\hline
\end{tabular}

FIG. 2 Image analysis results for different regions of interest (colors do not correspond between the EDS image and the image analysis colors). Each region of interest represents a gray level segment and corresponds with different elements. 